\title{
Cow Milk
}

National Cancer Institute

\section{Source}

National Cancer Institute. Cow Milk. NCI Thesaurus. Code C66153.

A body substance containing proteins, fats, lactose, and various vitamins and minerals produced by the mammary glands of mature female bovines. 